\section{Nobel Prizes: 2}

$\mathrm{A}^{\text {LTHOUGH the pioneer discoveries }}$ on tumour viruses by Peyton Rous were begun in 1911, it is only in the past two decades that the particular importance of these viruses has been recognised, not only as causes of cancer, but as vital keys to the structure and function of animal cells. We now know that a prime feature of these tumour viruses is their ability to exist as integral parts of the chromosomes of their hosts, carried indefinitely from generation to generation in tumour cells, sometimes in normal cells, and even in germ cells, from parent to offspring. It is not surprising that the 1975 Nobel Prize for Medicine should be awarded to David Baltimore, Renato Dulbecco and Howard Temin, the three scientists whose discoveries revealed a phenomenon with such wide implications.

There can be few research workers with such a sustained harvest of discoveries as Renato Dulbecco. After graduating in medicine in Italy, and a period of initiation into phage genetics with Salvador Luria, he moved to CalTech in 1952, and during the next few years, with Marguerite Vogt, his long standing collaborator, he set the scene for the whole new era of quantitative animal virology in cultured cells which has followed. This was begun by the development of the plaque assay, a precise and direct measurement of virus particles. making possible for the first time a detailed analysis of virus growth and virus antibody interaction, to say nothing of the isolation of genetically pure stocks of virus, an essential first step for the subsequent isolation of mutants. Much of this early work was with polio virus and provided the basis for the development of the live poliomyelitis vaccines.

In the late 1950s, however, interest in Dulbecco's laboratory was shifting to the tumour viruses, and it was here that Harry Rubin and Howard Temin (then a graduate student) first successfully applied Dulbecco's quantitative approach to the study of Rous sarcoma virus, by developing an assay of transformation, a cancer-like change induced by the virus in cultured cells. Meanwhile, Dulbecco and Vogt had concentrated on the recently discovered polyoma virus from mice, and in 1959 they reported transformation by this virus leading not only to abnormal growth of cells in culture, but also to the development of tumours when the cells were transplanted into animals. In the next few years, which included a sabbatical period in Glasgow, Dulbecco and his associates then went on to characterise the DNA which consituted the genome of the virus. It was found to be a small circular molecule, and this alone was sufficient to produce the neoplastic transformation.

The studies of transformation and tumour induction by polyoma virus, and the closely related SV40 virus of monkeys, soon posed a crucial question concerning the fate of the virus, and its role, once the initial neoplastic event had taken place in an individual cell. Did the virus simply hit and run, or did it continue to multiply and persist in all the progeny cells? If the latter, was it essential for the continued abnormal behaviour of these cells? It was soon shown by Dulbecco and Vogt that the presence and growth of virus particles were not essential features of the transformed state, but there were indications, from the work of Habel and others on new cell surface antigens for example, that viral coded information might be present in the cells. The ring structure of the viral DNA had, in fact, suggested to Dulbecco that it might be incorporated into the chromosomal DNA of the host in a manner similar to prophage in lysogenic bacteria.

It was in 1967 and 1968, after moving to the new Salk Institute as a Founder Fellow, that Dulbecco and his associates produced the first evidence that this was, indeed, the case. First with Watkins the presence of a complete set of viral genes was demonstrated in apparently virus-free transformed cells, by fusion with a second 'permissive' cell which released the block in virus growth. Then with Westphal, and later with Sambrook and Srinivsan, molecular hybridisation was used to show that viral nucleic acid sequences were not only present in the transformed cells, but were chemically attached to the chromosomal DNA of the host. This fundamental series of discoveries, and others that followed, showed how, after the initial transformation or tumour induction, the virus might persist and so affect the behaviour of all the descendents of the original cell.

To investigate the role of the tumour virus genes in the cells, Dulbecco had begun the study of conditional mutants of the viruses, first of all at CalTech with his student, Fried, who isolated the tsa mutant of polyoma virus, and subsequently with Vogt and Eckhart at the Salk Institute, on a whole series of new mutants. Finally, as if this were not enough, Dulbecco has produced major contributions on the physiology of the transformed cells and their normal counterparts, work which has actively continued since he moved to London in 1972.

The discovery that a DNA-containing virus could persist by integration of its genome in an animal cell was remarkable enough, but there was at least a model available in the integrated prophage of lysogenic bacteria. The RNA-containing tumour viruses were a different kettle of fish altogether-. or so it seemed. Rous sarcoma cells, or cells transformed in culture by this and similar viruses, may admittedly harbour virus particles, but since the viruses contain RNA alone there seemed to be no way in which the genetic information could be transferred into DNA to allow a stable relationship with the chromosomes of the host cells. Nevertheless, the virus-cell relationship was clearly very stable, and there were preliminary indications that some of the RNA tumour viruses could be transmitted in chromosomes from parent to offspring in Mendelian fashion. It was this problem which attracted Howard Temin.

Temin had continued his early work with Rous sarcoma virus, and using virus mutants made the interesting finding that the virus could determine cell morphology. After he left Dulbecco's laboratory he moved to the McArdle Institute at Madison, Wisconsin, and there began to study the nature of the provirus, as he called it, in transformed cells. After showing that DNA synthesis was a necessary requirement for transformation, he went on to search for viral DNA in the transformed cells. Soon came his scandalous proposal that the genetic information in tumour virus RNA was first transcribed into DNA, which was, in turn, incorporated in the transformed cells. This was not, as some have suggested, an offence to Crick's central dogma, but it was. none the less, pretty unorthordox. Indeed, when Temin's first nucleic acid hybridisation data, purporting to show Rous virus DNA sequences in transformed cells, were published in 1964, they were not very convincing and there was a good deal of sad head shaking. In time, Baluda produced more convincing evidence for viral DNA sequences in transformed cells which seemed to support Temin's views, but the princinal stumbling block was the absence of any known enzyme system which could make a DNA copy from an RNA molecule, and it was this problem which Temin began to investigate.

Meanwhile, in 1968 David Baltimore had come to work in Dulbecco's laboratory. While there, and later at MIT, he made a serics of important discoveries on the replicating form of the RNA of poliovirus, and on the processing of the newly synthesised virus proteins. He had not worked with tumour viruses, but was presumably influenced by the work going on around him in Dulbecco's laboratory, and by Baluda's recent hybridisation data. After he left to take up his position in 
Boston, he began, like Temin, to look for an enzyme in Rous sarcoma virus which would synthesise DNA on a RNA template.

And so it turned out that, simultaneously but quite independently in May 1970, Baltimore, and Temin with his colleague, Mizutani, reported the phenomenon which soon became known as reverse transcription. In elegantly simple experiments they both showed that radioactive precursors were incorporated into DNA in Rous sarcoma virus particles containing RNA alone, and this DNA turned out to be complementary to the virus RNA. Baltimore and his colleagues have since gone on to investigate in considerable detail the activity of reverse transcriptase, the enzyme concerned, and in a series of outstanding papers have described most of the steps by which double-stranded DNA is constructed from virus RNA.

Many of the implications of the discovery of reverse transcription were immediately obvious and were breathtaking in their scope. It immediately vindicated Temin's hypothesis that RNA tumour viruses persist in tumour cells in the form of DNA copies, and it was not long before Hill and Hillova demonstrated infectious DNA in Rous virus-transformed cells. This linked the work of Baltimore and Temin to that of Dulbecco, and permitted a unified view of tumour viruses in general, since they are clearly all DNA viruses at heart, at least in their stage of association with the host cells. In addition, the discovery of reverse transcriptase has itself provided an extraordinarily powerful new tool, since it makes possible the construction of highly labelled complementary DNA molecules which can be used as probes to search for similar sequences in either DNA or RNA. This has led, not to new and sensitive methods in the search for human tumour viruses, particularly those developed by Spiegelman, Gallo, and others, but also to more general studies on messenger RNA and its relationship to the chromosomal DNA, for example, in studies on the haemoglobin and $\gamma$ globulin genes. Finally, the demonstration that the flow of genetic information from DNA to RNA can be reversed has provoked a whole range of new and exciting speculations (and even experiments) on the relationships of viruses and their hosts, not least with Temin's own provoking ideas about the 'normal' role of protoviruses in gene amplification and differentiation.

The discoveries by this year's prizewinners have opened entirely new prospects in virology and cancer studies, and have added a quite new dimension to molecular biology. Had he survived, Peyton Rous, who started it all and who waited 55 years for his Nobel Prize, would have been the first to express his delight at the Committee's choice.

M. G. P. Stoker

\section{international news}

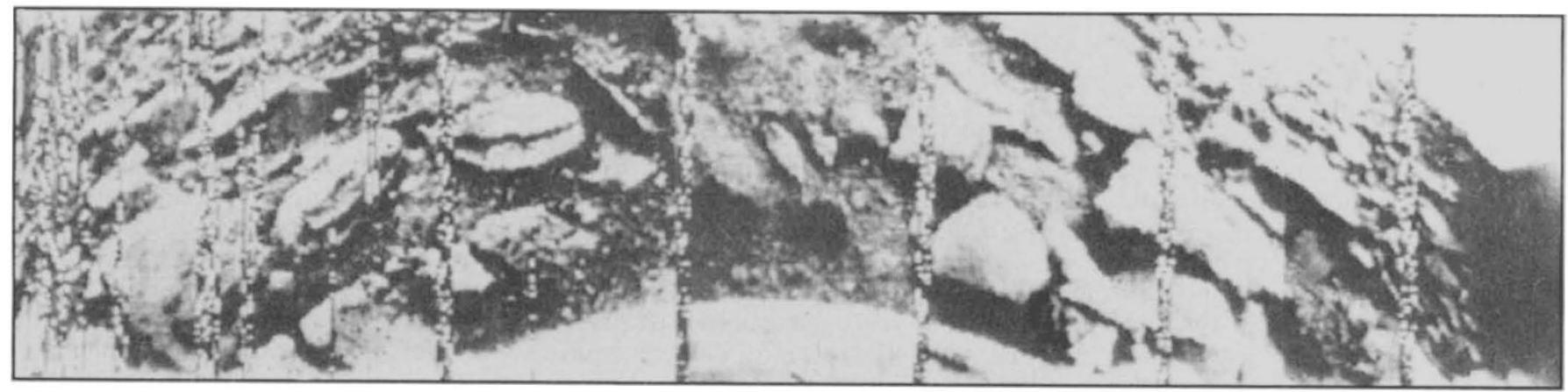

THE two Venus probes, Venera 9 and 10 , which soft-landed successfully on October 22 and 25, respectively, at sites some $2,200 \mathrm{~km}$ apart, have resulted in considerable rethinking of many ideas about the planet. The members of the Soviet Venus project, faced with pictures of sharp, angular, granitelike rocks (see picture above) instead of the expected sand, have been quick to comment. The sharp, "young appearance of the rocks" said mineralogist Aleksandr Vasilevskii, indicates recent seismic or volcanic activity, typical of a "live" planet. The sharp shadows, indicative of sunlight filtering through the cloud cover to the surface and the flat landscape, rather than the expected "gold-fish bowl effect" produced by postulated atmospheric distortion, have considerably opened up the possibilities of further visual observation of the surface, since it is now clear that the

\section{Venus, courtesy of Moscow}

\author{
from Vera Rich
}

photographs obtained can be considered and interpreted as received, without the need for empirical correction and compensation. M. Ya. Marov, of the LongRange Space Communications Centre, spoke of the photographs as inaugurating a new era in space research, in which "clearly directed complex investigations" would replace the somewhat hit-and-miss efforts of the previous "age of reconnaisance". In the same mood of euphoria, Pravda also speculated on the possibility of manned Venus missions in the next century.

Meanwhile, the remaining data gathered and transmitted by the probes, including analysis of the cloud cover and the optical characteristics of the atmosphere, has gained relatively little attention, and must await routine processing.

- The Soviet Minister of Education, Mr V. P. Elyutin, visited Britain and, more particularly, the Open University last week. This visit, clearly an exercise in detente, yielded little definite outcome, although some fields of cooperation between the Open University and its Soviet opposite number are envisaged. $\mathrm{Mr}$ Elyutin spoke of the possible mutual publication of materials, and mutual production of television programmes, and also joint research work on common problems such as environmental protection and also special research into the theoretical problems of higher education.

Speaking of Soviet higher education, 